\title{
Lactobacillus salivarius BP121 prevents cisplatin-induced acute kidney injury by inhibition of uremic toxins such as indoxyl sulfate and $p$-cresol sulfate via alleviating dysbiosis
}

\author{
TAE-HEE LEE ${ }^{1 *}$, DONGSUN PARK ${ }^{2 *}$, YANG JI KIM $^{1,3}$, ISAAC LEE ${ }^{4}$, SUAE KIM $^{5}$, CHANG-TAEK OH $^{5}$, \\ JOEM-YONG KIM ${ }^{5}$, JIHYUN YANG ${ }^{6}$ and SANG-KYUNG JO ${ }^{6}$
}

\begin{abstract}
${ }^{1}$ Department of Toxicity Evaluation, Centralbio Co., Ltd., Gimpo, Gyeonggi 31499; ${ }^{2}$ Department of Biology Education, Korea National University of Education, Cheongju, North Chungcheong 28173; ${ }^{3}$ Department of Toxicity Evaluation, Konyang University, Daejeon 35365; ${ }^{4}$ Hoseo Toxicological Research Center, Hoseo University, Asan, Chungnam 31499;

${ }^{5}$ Research Institute, Research and Development Center, Green Cross WellBeing Corporation, Seongnam, Gyeonggi 13595; ${ }^{6}$ Department of Internal Medicine, Division of Nephrology, Korea University Medical College, Seoul 02841, Republic of Korea
\end{abstract}

Received July 12, 2019; Accepted December 24, 2019

DOI: $10.3892 / \mathrm{ijmm} .2020 .4495$

\begin{abstract}
The gut microbiota is important for maintaining the integrity of the intestinal barrier, promoting immunological tolerance and carrying out metabolic activities that have not evolved in hosts. Intestinal dysbiosis is associated with chronic kidney disease and probiotic supplementation has been shown to be beneficial. However, it is not known whether gut microorganisms-specifically, lactic acid bacteria (LAB) can protect against acute kidney injury (AKI). To address this issue, the present study investigated the effects of Lactobacillus salivarius BP121, an intestinal LAB isolated from the feces of newborns, in a rat model of cisplatin-induced AKI and also in Caco-2 human intestinal epithelial cells. BP121 prevented cisplatin-induced AKI in rats, as demonstrated by decreases in inflammation and oxidative stress in kidney tissue and in serum levels of uremic toxins such as indoxyl sulfate (IS) and $p$-cresol sulfate (PCS). BP121 also reduced intestinal permeability, as determined using fluorescein isothiocyanate-dextran by immunohistochemical detection of tight junction (TJ) proteins such as zona occludens-1 and occludin. The abundance of Lactobacillus spp., which are beneficial intestinal flora, was increased by BP121; this was accompanied by an increase in the concentrations of short-chain fatty acids in feces. Additionally, $\mathrm{H}_{2} \mathrm{O}_{2}$-induced TJ protein damage was
\end{abstract}

Correspondence to: Professor Sang-Kyung Jo, Department of Internal Medicine, Division of Nephrology, Korea University Medical College, 73 Inchon-ro, Seongbuk-gu, Seoul 02841, Republic of Korea

E-mail: sang-kyung@korea.ac.kr

${ }^{*}$ Contributed equally

Key words: acute kidney injury, cis-diammineplatinum (II) dichloride, lactic acid bacteria, dysbiosis, indoxyl sulfate, $p$-cresol sulfate reduced in Caco-2 cells treated with BP121 culture supernatant, an effect that was reversed by the 5' AMP-activated protein kinase (AMPK) inhibitor Compound $\mathrm{C}$ and Toll-like receptor (TLR)4 inhibitor TLR4-IN-C34. In conclusion, this study demonstrated that $L$. salivarius BP121 protects against cisplatin-induced AKI by decreasing inflammation and oxidative stress and this renoprotective effect is partially mediated by modulating the gut environment and thereby suppressing IS and PCS production as well as by regulating AMPK and TLR4 dependent TJ assembly.

\section{Introduction}

Acute kidney injury (AKI) is a clinical syndrome characterized by a rapid decrease in renal function accompanied by accumulation of waste products such as urea $(1,2)$. The incidences of non-dialysis-requiring and dialysis-requiring AKI in the U.S. are 522 and 29.5 per 100,000 people/years, respectively (3). Drug-induced AKI is a common occurrence in clinical practice because the kidney is a major target of various toxic chemicals $(4,5)$. Although the incidence is difficult to estimate due to variable patient characteristics, many newly developed drugs have distinct mechanisms of nephrotoxicity, making it the second most common cause of hospital-acquired AKI (4). Recent studies have revealed a close relationship between the gut microbiome and various kidney diseases; however, the association between intestinal bacteria and nephrotoxic AKI remains unclear $(6,7)$.

Cis-diammineplatinum (II) dichloride (cisplatin) is an inorganic platinum-based chemotherapy drug that is widely used in the treatment of solid tumors including testicular, ovarian, cervical and head and neck cancers $(5,8)$. However, nephrotoxicity is major side effect of cisplatin $(9,10)$, which binds to DNA to form inter- and intra-stand crosslinks that damage DNA templates or inhibit DNA synthesis and replication. The potential for damage is even greater in rapidly dividing cells such as cancer or epithelial cells (10); however, it can also disrupt the intestinal epithelium, thereby increasing 
intestinal permeability and causing systemic inflammation by permitting the egress of microbes and their toxins $(11,12)$.

Probiotics are living, non-pathogenic microbes (bacteria or yeast) that provide beneficial nutrients to the host $(13,14)$. Probiotics can directly inhibit the growth or promote death of pathogenic microorganisms by producing anti-microbial substances such as short-chain fatty acids (SCFAs) and have shown immunomodulatory effects in several chronic inflammatory disease models. L. salivarius BP121 (BP121) that was isolated from infant feces. L. salivarius has been shown to have remarkable probiotic efficacy because it had high survival rate in gastrointestinal tract conditions and produced antimicrobial compounds such as SCFAs (15). Previous studies have shown that L. salivarius produces abundant propionate/butyrate $(16,17)$. Additionally, in previous studies, improved barrier function by SCFA was reported (18-20). SCFAs such as butyrate and acetate are known to protect intestinal barrier integrity by regulating through the activation of AMPK and TLR4. To date, there have been no studies investigating the possible benefits of probiotics including lactic acid bacteria (LAB) BP121 in the context of AKI.

AST-120 is an oral spherical carbon absorbent consisting of oral urea toxin remover and porous carbon particles. AST-120 reduces the accumulation of indoxyl sulfate in patients by adsorbing indole, a precursor of toxin sulfate. This function has the effect of slowing the progress of kidney disease (21).

To address this issue, the effect of BP121 treatment on a rat model of cisplatin-induced AKI was investigated, as well as the mechanistic basis for the observed effects of BP121 using Caco-2 human intestinal epithelial cells.

\section{Materials and methods}

Isolation and selection of strains from infant feces. LAB were isolated from fresh feces of new born infants (22). The samples were blended with $50 \mathrm{ml} 0.85 \% \mathrm{NaCl}$ solution and serially diluted 10 -fold in $0.85 \% \mathrm{NaCl}$; each diluted solution was plated onto De Man, Rogosa, and Sharpe (MRS) agar (BD Biosciences; Becton, Dickinson and Company). The plates were incubated in an anaerobic jar with Anaeropack (BD Biosciences; Becton, Dickinson and Company) at $37^{\circ} \mathrm{C}$ for $72 \mathrm{~h}$. White colonies on the plates were individually picked and streaked on fresh MRS agar plates. Isolated strains were identified based on the near-complete $16 \mathrm{~S}$ rRNA gene sequence using the EZ-taxon server (http://www.ezbiocloud.net).

$L A B$ screen for indoxyl sulfate (IS) inhibitory activity. LAB strains were screened for their ability to reduce IS levels as previously described (23). The strains were incubated in MRS broth with $60 \mu \mathrm{g} / \mathrm{ml}$ IS and the one with the highest inhibitory activity was selected. After incubation for $72 \mathrm{~h}$, IS levels were measured with the Indican Assay kit (Sigma-Aldrich; Merck $\mathrm{KGaA})$.

Analysis of SCFA in the probiotics conditioned medium and stool. L. salivarius BP121, L.acidophilus BP105 and L. rhamnosus GG were used for analysis of SCFA in conditioned medium. A total of three strains were incubated in MRS broth at $37^{\circ} \mathrm{C}$ for $48 \mathrm{~h}$. SCFA analyzed using a high-performance liquid chromatography (HPLC; Hewlett-Packard HP1100 series; Agilent Technologies, Inc.), equipped with a UV-vis detector and diode array detector. (Colum; YMC-Pack FA 250/6 mm I.D., Mobile phase; acetonitrile:water (85:15), pH 4.5 adjusted by $0.1 \%$ trifluoro acetic acid; flow rate, $1.2 \mathrm{ml} / \mathrm{min}$, Temperature $35 \mathrm{UV}$ at $400 \mathrm{~nm}$ ). Culture medium was directly injected into the chromatograph after filtering with $0.2 \mathrm{um}$ syringe filter. Also, $200 \mathrm{mg}$ stool was mixed with $0.15 \mathrm{mM}$ $\mathrm{HCIO}_{4}$ and then sonicated at $37^{\circ} \mathrm{C}$ and $70 \mathrm{KHz}$ for $15 \mathrm{~min}$. The prepared samples were filtered with $0.2 \mu \mathrm{m}$ syringe filter. The filtered sample $20 \mu \mathrm{l}$ was injected into the HPLC. Acetate, propionate and butyrate mixture were used to prepare a standard curve (range of 1-1,000 ppm).

Animals and cell line. A total of 48 male Sprague-Dawley rats (SantacoBioKorea, Inc.) weighing 187-221 g (8 weeks old) were housed two rats per cage in a room with controlled temperature $\left(20-24^{\circ} \mathrm{C}\right)$ and humidity $(40-70 \%)$ on a 12 -h light/dark cycle with free access to food and sterile water. Experimental procedures involving the rats were approved by the Institutional Animal Care and Use Committee of Hoseo University [appr oval no. HSIACUC-18-097(1)]. Caco-2 cells were purchased from the Korean Cell Line Bank (cat. no. 30037.1) and were maintained as a monolayer in minimal essential medium (Thermo Fisher Scientific, Inc.) supplemented with $10 \%$ fetal bovine serum (Thermo Fisher Scientific, Inc.), $25 \mathrm{mM}$ HEPES, and $25 \mathrm{mM} \mathrm{NaHCO}_{3}$ at $37^{\circ} \mathrm{C}$ in a humidified atmosphere of $5 \% \mathrm{CO}_{2}$.

Experimental design. The 48 rats were randomly divided into six groups ( $\mathrm{n}=8$ each). The AKI model was established by single intraperitoneal (i.p.) injection of cisplatin $(8 \mathrm{mg} / \mathrm{kg}$ body weight in $10 \mathrm{ml}$ saline). BP121 was administered daily by oral gavage [i.e., per os (p.o.) administration] starting from 10 days prior to and continuing until 4 days after cisplatin injection. Kremezin fine granules (AST120; CJ Corp.) were orally administered at $3.6 \mathrm{~g} / \mathrm{kg}$ body weight for 4 days after cisplatin injection. The groups were as follows: Normal control, saline (i.p.) + saline (p.o.); cisplatin, cisplatin (i.p.) + saline (p.o.); BP121-L, cisplatin (i.p.) + BP121 1x10 (p.o.); BP121-M, cisplatin (i.p.) + BP121 $1 \times 10^{9}$ (p.o.); BP121-H, cisplatin (i.p.) + BP121 1x10 ${ }^{10}$ (p.o.), AST120, cisplatin (i.p.) + AST120 (p.o.; Fig. 1). BP121 was obtained from. Green Cross Wellbeing Corp., (Fig. 1).

Intestinal permeability. Intestinal permeability was measured by administering fluorescein isothiocyanate-labeled dextran (60 mg/kg body weight, MW = 4,000 kDa; Sigma-Aldrich; Merck $\mathrm{KGaA}$ ) to rats by oral gavage on day 4 after cisplatin injection. At $4 \mathrm{~h}$ after gavage, $0.6 \mathrm{ml}$ of blood was drawn via the jugular vein and the fluorescence intensity of the plasma was measured using a GloMax-Multi+ fluorophotometer (excitation/emission, 490/525 nm; Promega Corporation).

Histological analysis. Kidney and small intestine tissues were fixed at room temperature for $48 \mathrm{~h}$ in $10 \%$ neutral buffered formalin, embedded in paraffin, and sectioned at a thickness of $4 \mu \mathrm{m}$. Kidney tissue sections were stained at room temperature with periodic acid-Schiff (PAS) (periodic acid for $5 \mathrm{~min}$ and Schiff reagent for $15 \mathrm{~min}$ ) and observed under a light microscope. The staining intensity (reflecting the area of tissue damage) was assessed in five randomly selected areas at x200 magnification as follows: None (score: 0$)=$ normal, 


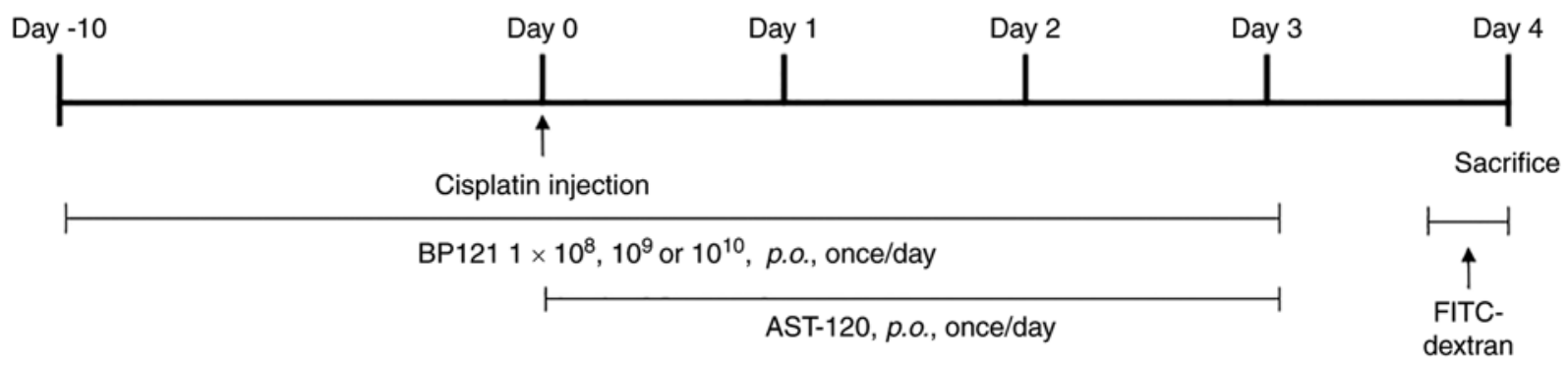

Figure 1. Diagram of the study design. P.o., per os; FITC, fluorescein isothiocyanate.
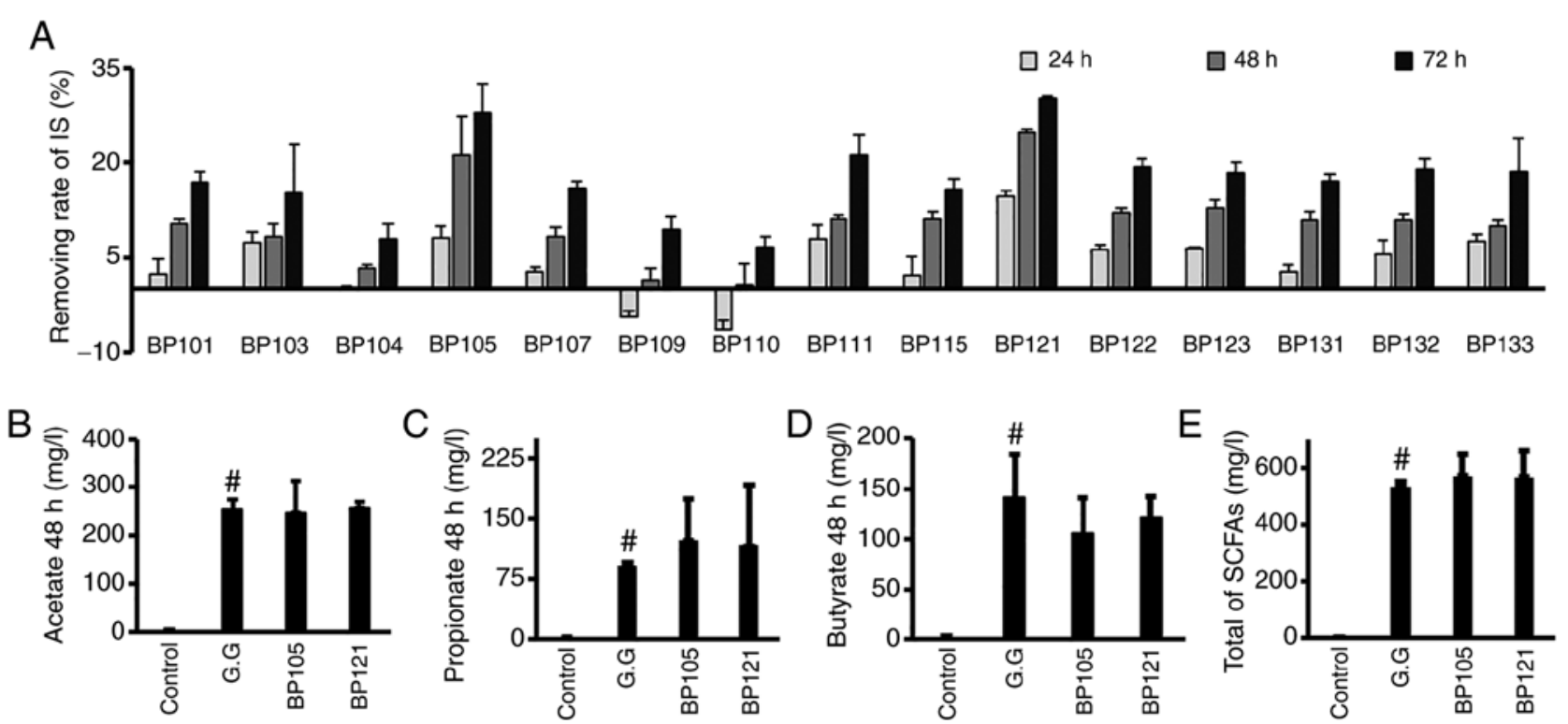

Figure 2. Effect of LAB strains on IS inhibiting effects and comparison of SCFA production capacity between Lactobacillus salivarius BP121, Lactobacillus acidophilus BP105 and L. rhamnosus GG. (A) Rate of IS removal. (B) Acetate, (C) propionate, (D) butyrate and (E) total of SCFAs. SCFA, short chain fatty acids. "P<0.05, normal vs. Lactobacillus rhamnosus GG.

weak (score: 1$)=<10 \%$, mild (score: 2$)=10-25 \%$, moderate $($ score: 3$)=26-75 \%$ and strong $($ score: 4$)=>75 \%(24)$. Immunohistochemical detection of monocyte chemoattractant protein (MCP)1, B cell lymphoma 2-associated X protein (Bax) in kidneys, zona occludins (ZO)-1, and occludin in small intestine was performed using a commercial kit (Abcam; cat. no. ab80436) and antibodies [all from Abcam; cat. nos. ab9668 (1:500), ab32503 (1:250), ab96587 (1:500) and ab216327 (1:500), respectively]. Apoptotic cells were detected with the terminal deoxynucleotidyl transferase dUTP nick end labeling (TUNEL) assay using the TACS 2 TdT DAB (diaminobenzidine) kit (Trevigen, Inc.; cat. no. 4810-30-k) according to manufacturer's protocol. The average positive cell number was calculated by observing 10 fields at x 200 magnification using a light microscope.

Biochemical parameters. Blood samples were collected from rats and serum levels of blood urea nitrogen (BUN), creatinine, and C-reactive protein (CRP) were measured with a Model 7020 automated biochemistry analyzer (Hitachi, Ltd.). IS and $p$-cresol sulfate (PCS) concentrations in serum were analyzed by liquid chromatography (ACQuity ${ }^{\mathrm{TM}}$ ) followed by tandem mass spectrometry (TSQ Quantum Ultra; Thermo Fisher Scientific, Inc.) (Data SI).
Evaluation of oxidative stress and inflammation. A 10-ml volume of phosphate-buffered saline was added to $0.5 \mathrm{~g}$ kidney tissue cut into 1-2 $\mathrm{mm}$ pieces and homogenized. The homogenate was centrifuged at $5,000 \mathrm{xg}$ for $10 \mathrm{~min}$ at $4^{\circ} \mathrm{C}$; the supernatant was collected and the levels of interleukin (IL)-6 (cat. no. MBS824560) and tumor necrosis factor (TNF)- $\alpha$ (cat. no. MBS175904) (both from MyBioSource); malondialdehyde (MDA; Cayman Chemical; cat. no. 10009055); and reduced to oxidized glutathione ratio (GSH/GSSG; Abcam; cat. no. ab138881) were analyzed.

Analysis of gut microbiota abundance. Fecal samples for gut microbiome profiling were collected at 4 days after cisplatin injection and stored at $-70^{\circ} \mathrm{C}$ until analysis. Species were identified by $16 \mathrm{~S}$ rRNA pyrosequecing using the EzTaxon database at Chunlab of Seoul National University (Seoul, South Korea). The procedure included sorting and quality pre-screening, trimming of primer sequences, schematic structure assignment, and chimera testing.

Effect of BP121 on tight junction (TJ) regulation in Caco-2 cells. Caco-2 cells were treated with $20 \mu \mathrm{M} \mathrm{H}_{2} \mathrm{O}_{2}$ (Volatile Free Acid Mix; Sigma-Aldrich; Merck KGaA; cat. no. CRM46975) to cause TJ protein damage; the culture supernatant of 

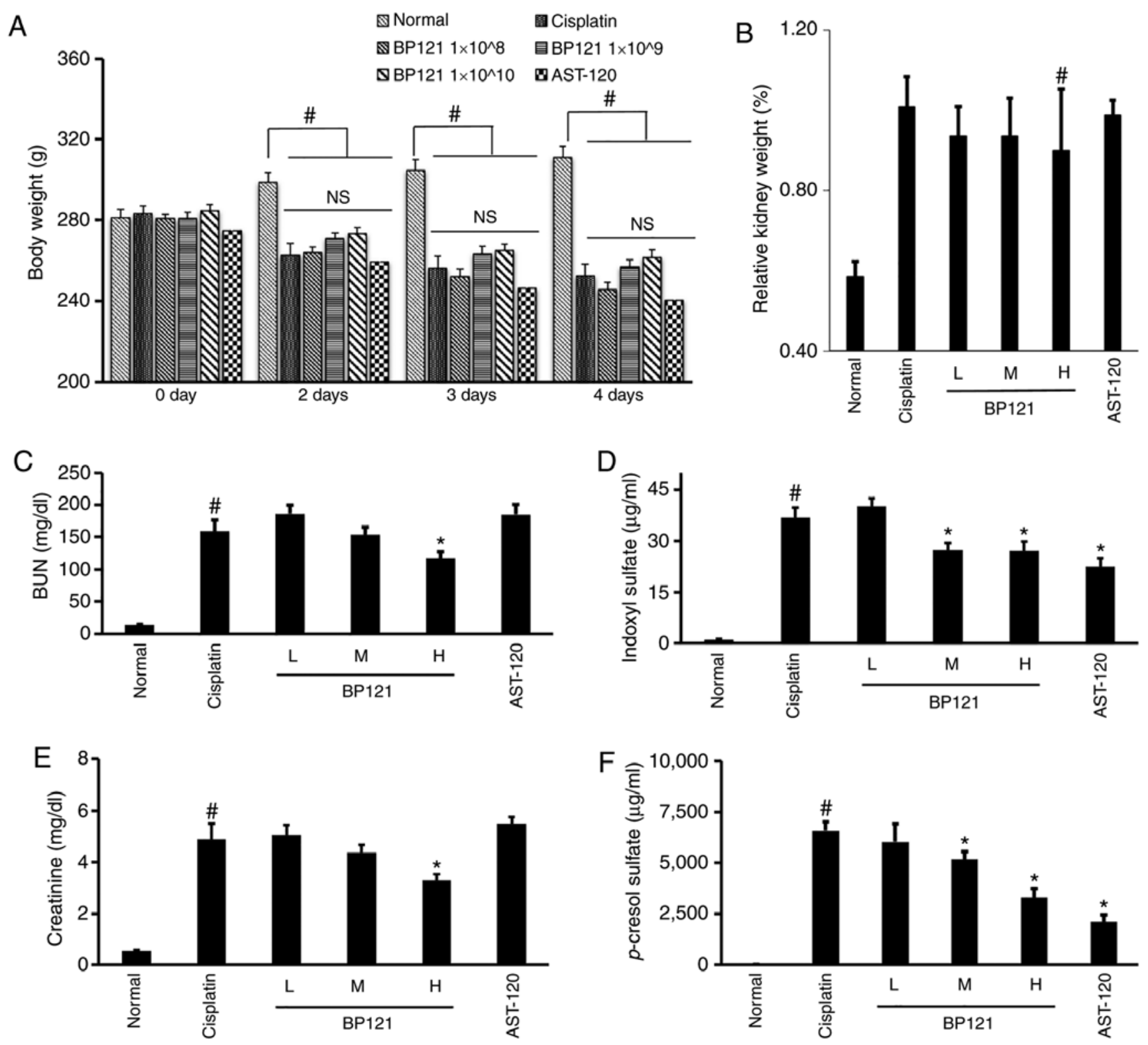

Figure 3. Physiological parameters in rats with cisplatin-induced AKI. Changes in (A) body weight and (B) relative kidney weight. Concentration of the urinary toxins (C) BUN, (D) IS, (E) CREA and (F) $p$-cresol sulfate in rats with cisplatin-induced AKI. L = BP121 1x10 ${ }^{8}, \mathrm{M}=\mathrm{BP} 1211 \times 10^{9}, \mathrm{H}=\mathrm{BP} 1211 \times 10^{10} .{ }^{\#} \mathrm{P}<0.05$, normal vs. cisplatin; ${ }^{\prime} \mathrm{P}<0.05$, cisplatin vs. treatment group. AKI, acute kidney injury; BUN, blood urea nitrogen; IS, indoxyl sulfate; CREA, creatinine; NS, not significant.

BP121 was used as a control. The 5' AMP-activated protein kinase (AMPK) inhibitor Compound C (cat. no. P5499) and the Toll-like receptor (TLR)4 inhibitor TLR-IN-C34 (cat. no. SML0832; both from Sigma-Aldrich; Merck KGaA) were used to evaluate the mechanism of $\mathrm{TJ}$ protein regulation. Each inhibitor was applied $30 \mathrm{~min}$ after $\mathrm{H}_{2} \mathrm{O}_{2}$ treatment. SCFAs and culture medium were added simultaneously with $\mathrm{H}_{2} \mathrm{O}_{2}$. ZO-1 and occludin were detected by immunofluorescence after incubation at $37^{\circ} \mathrm{C}$ for $24 \mathrm{~h}$; Alexa Fluor 488 (cat. no. ab150077; 1:400) and Alexa Fluor 647 (cat. no. ab150079; 1:400) (both from Abcam) were used as secondary antibodies and nuclei were stained with DAPI at $37^{\circ} \mathrm{C}$ for $15 \mathrm{~min}$ (Sigma-Aldrich; Merck KGaA; cat. no. D9542).

Statistical analysis. Data are expressed as the mean \pm standard error. Data were analyzed using SPSS (version 21.0;
IBM Corp.) software. After homogeneity of variances using Levene's test, one way ANOVA analysis was performed to evaluate the significant differences, with Tukey's post-hoc test. $\mathrm{P}<0.05$ was considered to indicate a statistically significant difference. Experiments were repeated 5-6 times for the in vivo investigations, or 3 times for the in vitro investigations.

\section{Results}

Isolation and selection of strains from infant feces. To screen for IS-inhibiting bacteria, bacteria obtained from fresh feces of newborn infants were grown on MRS agar plates. A total of 15 strains of LAB were selected and cultured in MRS broth with IS for $72 \mathrm{~h}$; the strain with the highest inhibitory effect on IS was found to be BP121. This strain was able to reduce the levels of IS by $>30 \%$ after $72 \mathrm{~h}$. To identify this strain, analysis of 
A

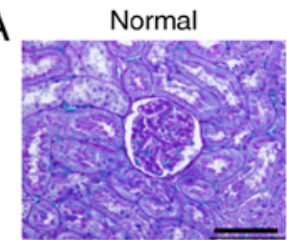

BP121 M

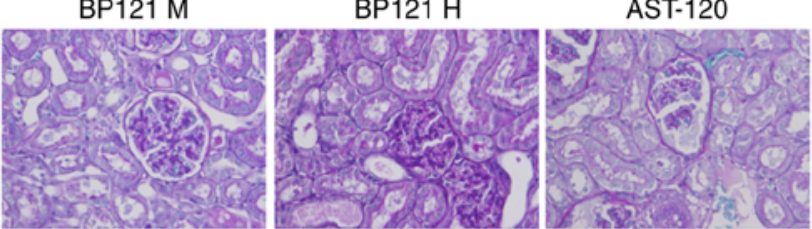

C

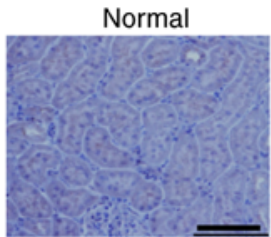

BP121 M

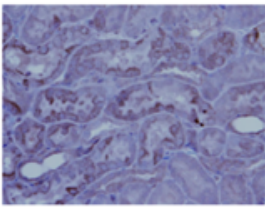

E
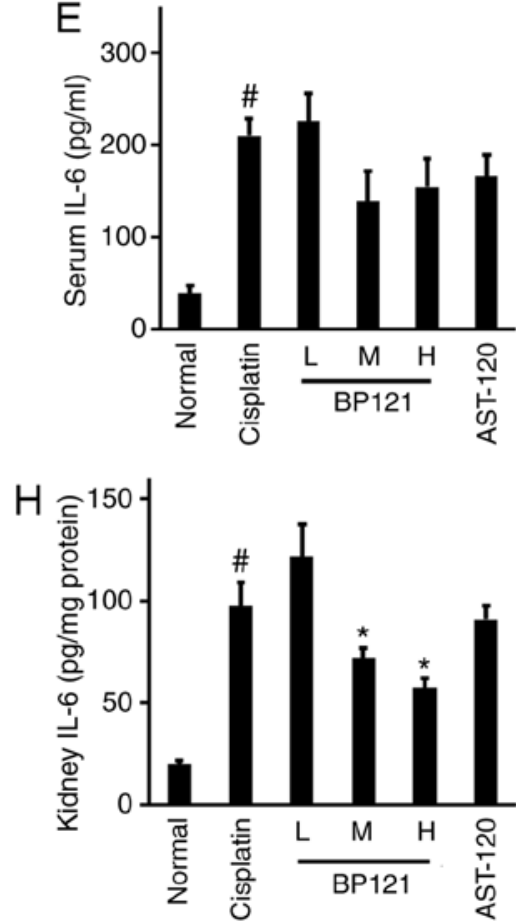

Cisplatin

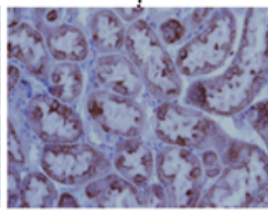

BP121 H

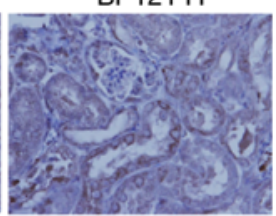

\section{$F^{\text {row }}$}
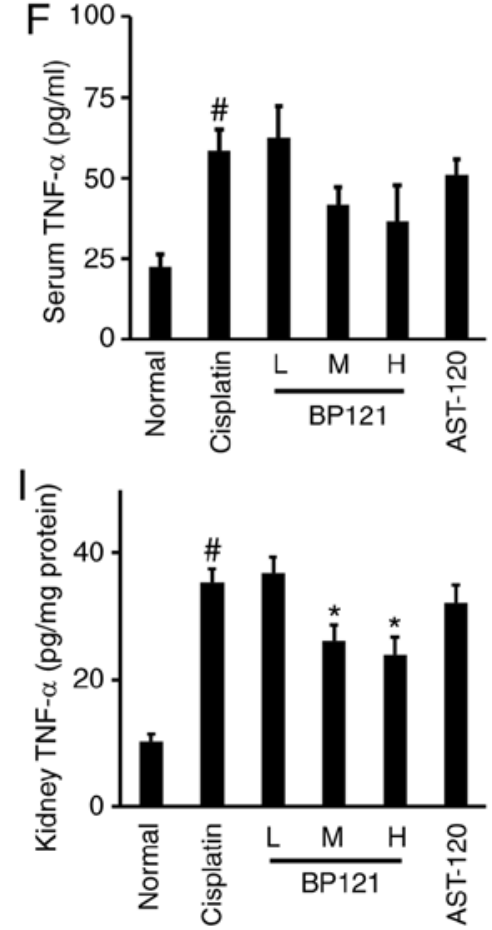

BP121 L

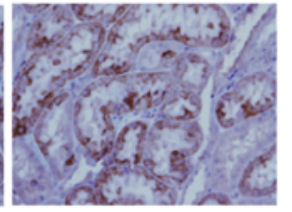

AST-120

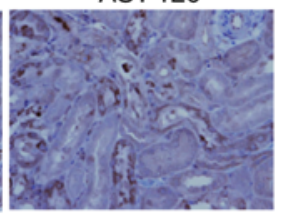

D
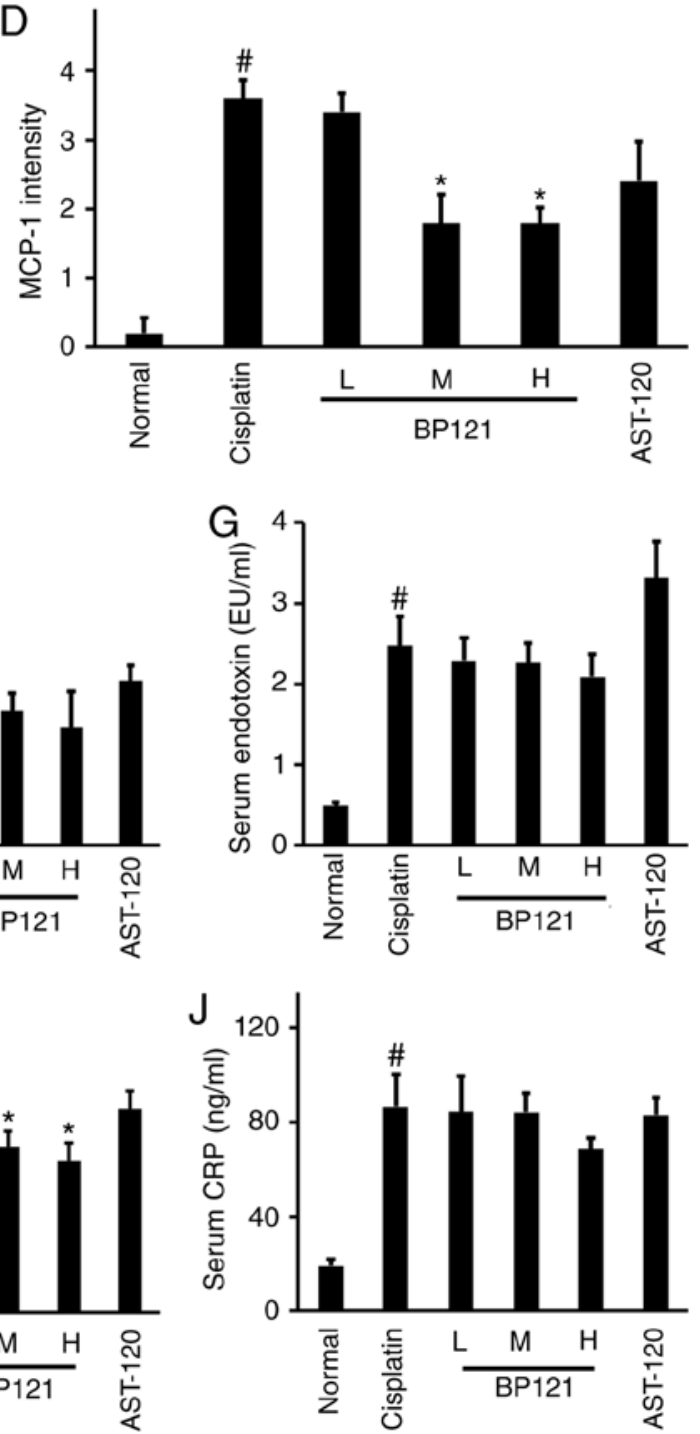

Figure 4. Histological analysis of the kidney and IL-6, TNF- $\alpha$, and CRP expression following cisplatin-induced AKI. (A) PAS staining and (B) injury score. (C) MCP-1 immunoreactivity and (D) intensity. Concentrations of (E) serum IL-6, (F) TNF- $\alpha$, (G) serum endotoxin, (H) kidney IL-6, (I) kidney TNF- $\alpha$ and (J) CRP levels were measured by ELISA. Scale bar $=100 \mu \mathrm{m}$. ${ }^{*} \mathrm{P}<0.05$, normal vs. cisplatin; ${ }^{*} \mathrm{P}<0.05$, cisplatin vs. treatment group. IL, interleukin; TNF, tumor necrosis factor; CRP, c-reactive protein; PAS, periodic acid Schiff; AKI, acute kidney injury.

the 16S rRNA gene sequence of BP121 (Table SI) revealed that it is closely related to Lactobacillus salivarius JCM1231, with 99.93\% sequence similarity. After culturing BP121 and BP105 for $48 \mathrm{~h}$, SCFA production was analyzed. Both strains produced SCFA similar to L. rhamnosus GG (Fig. 2 B-E). Based on these results, BP121 was selected as the test strain, which showed the highest IS inhibitory effect and high SCFA production (Fig. 2).

BP121 restores kidney weight and reduces serum urinary toxin levels. Rats that were administered BP121 did not show 


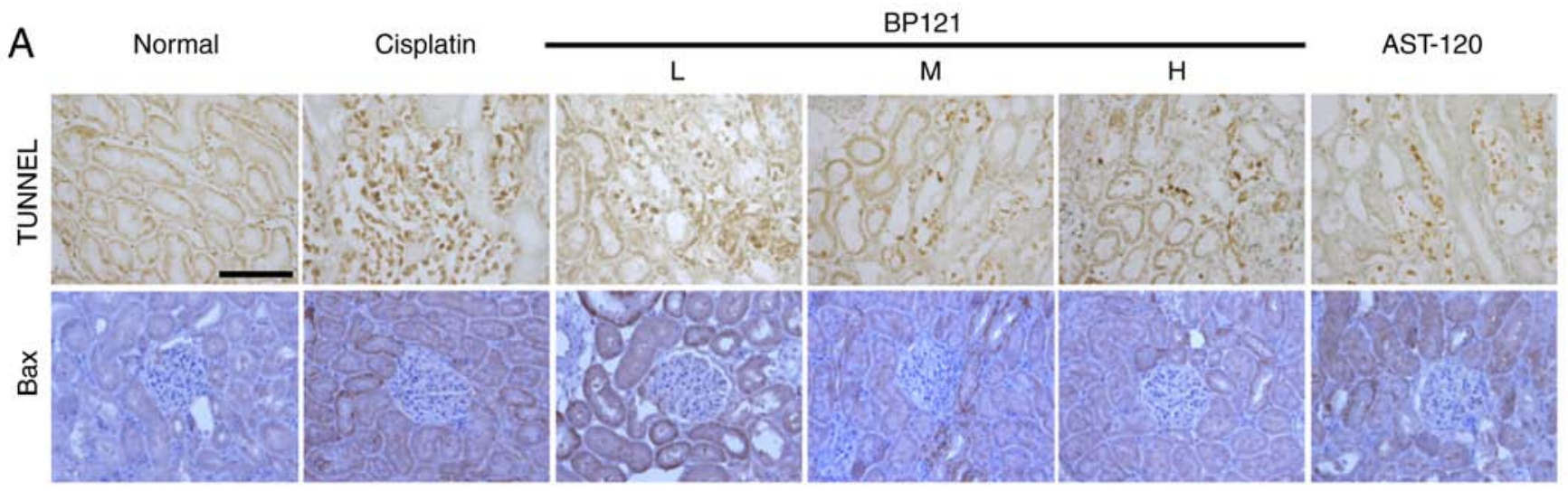

B

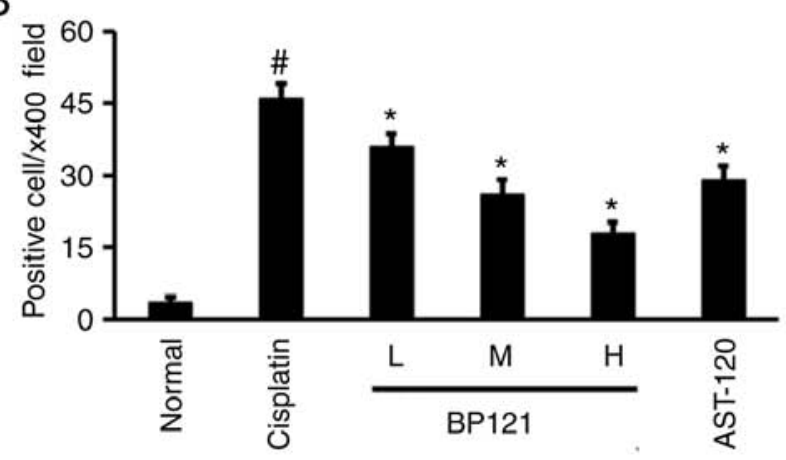

D

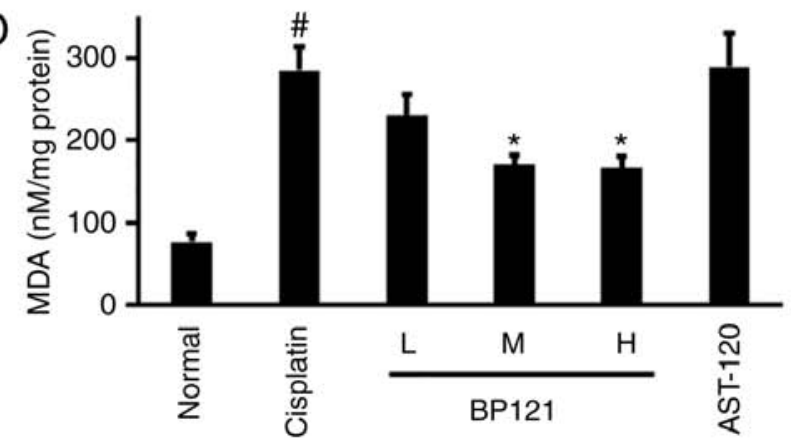

C
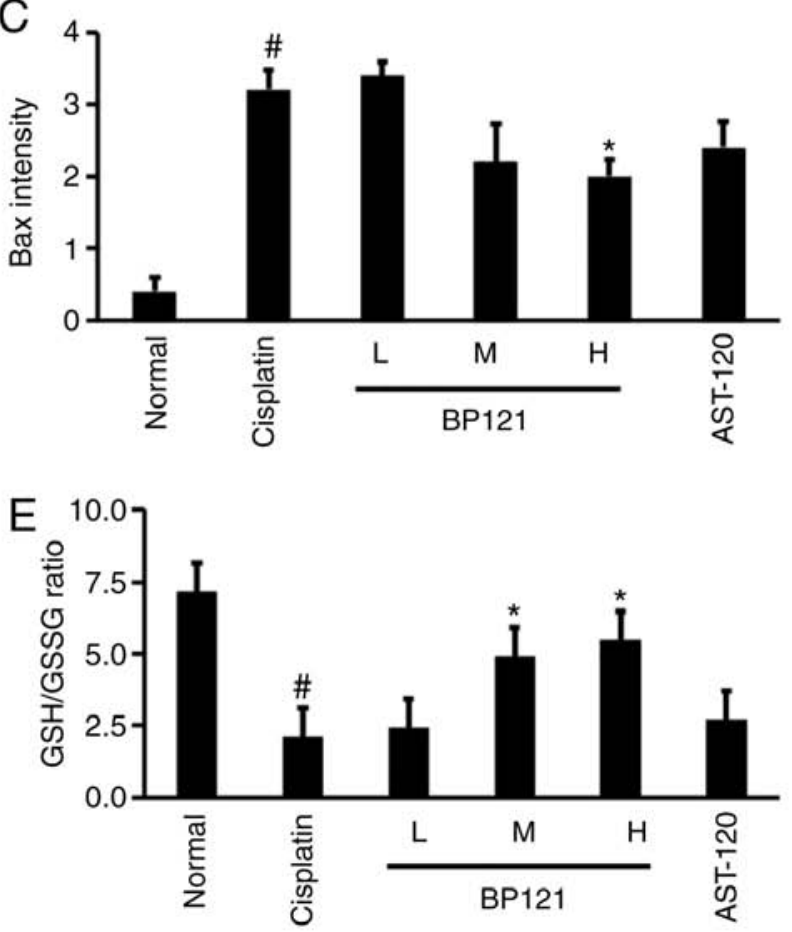

Figure 5. Histological analysis of kidney in rats with cisplatin-induced acute kidney injury. (A) TUNEL results are shown as (B) the number of positive cells per field at magnification, $\mathrm{x} 400$. (C) Bax immunolabeling. (D) MDA level and (E) GSH/GSSG ratio in kidney. Scale bar=100 $\mu$ m. ${ }^{\#} \mathrm{P}<0.05$, normal vs. cisplatin; ${ }^{*} \mathrm{P}<0.05$, cisplatin vs. treatment group. MDA, malondialdehyde.

any difference in body weight compared with those that were treated with cisplatin (Fig. 3A). On the other hand, the increase of relative kidney weight in cisplatin treated rats was partially decreased in rats who were administered a high dose of BP121 (Fig. 3B). Similar trends were observed for serum BUN and creatinine levels (Fig. 3C and E). In addition, BP121 caused a dose-dependent reduction in IS and PCS concentrations (Fig. 3D and F). AST-120 had no effect on body weight, relative kidney weight, or blood BUN and creatinine levels, but significantly reduced IS and PCS levels in the blood (Fig. 3).

BP121 suppresses inflammation and injury in kidney. PAS stained kidney tissue revealed extensive renal damage following cisplatin treatment; this was reduced by BP121-H administration (Fig. 4A and B). In addition, the increase in the number of MCP-1-positive cells induced by cisplatin was reversed by BP121 (Fig. 4C and D). Although there were no differences in serum levels of IL-6, TNF- $\alpha$, endotoxin and CRP between the cisplatin- and BP121-treated groups (Fig. 4E, G and J), IL-6 and TNF- $\alpha$ concentrations in kidney tissue homogenate were significantly reduced by BP121 treatment (Fig. 4H and I). Despite a substantial decrease of both IS and PC, AST-120 failed to show a renal protective effect (Fig. 4A-I).

BP121 inhibits apoptosis and oxidative stress in the kidney. The number of TUNEL positive apoptotic cells significantly increased in cisplatin induced AKI and BP121 treatment dose dependently reduced apoptosis.

A similar trend was observed in the expression of the pro-apoptotic protein Bax, which was upregulated in the kidney of cisplatin-treated rats and downregulated upon administration of a high dose BP121 (Fig. 5A-C). The MDA level and GSH/GSSG ratio were also measured as indices of oxidative damage and found that lipid peroxidation (MDA level) was increased whereas GSH/GSSG ratio was decreased by cisplatin injection; these changes were partially reversed by pretreatment 


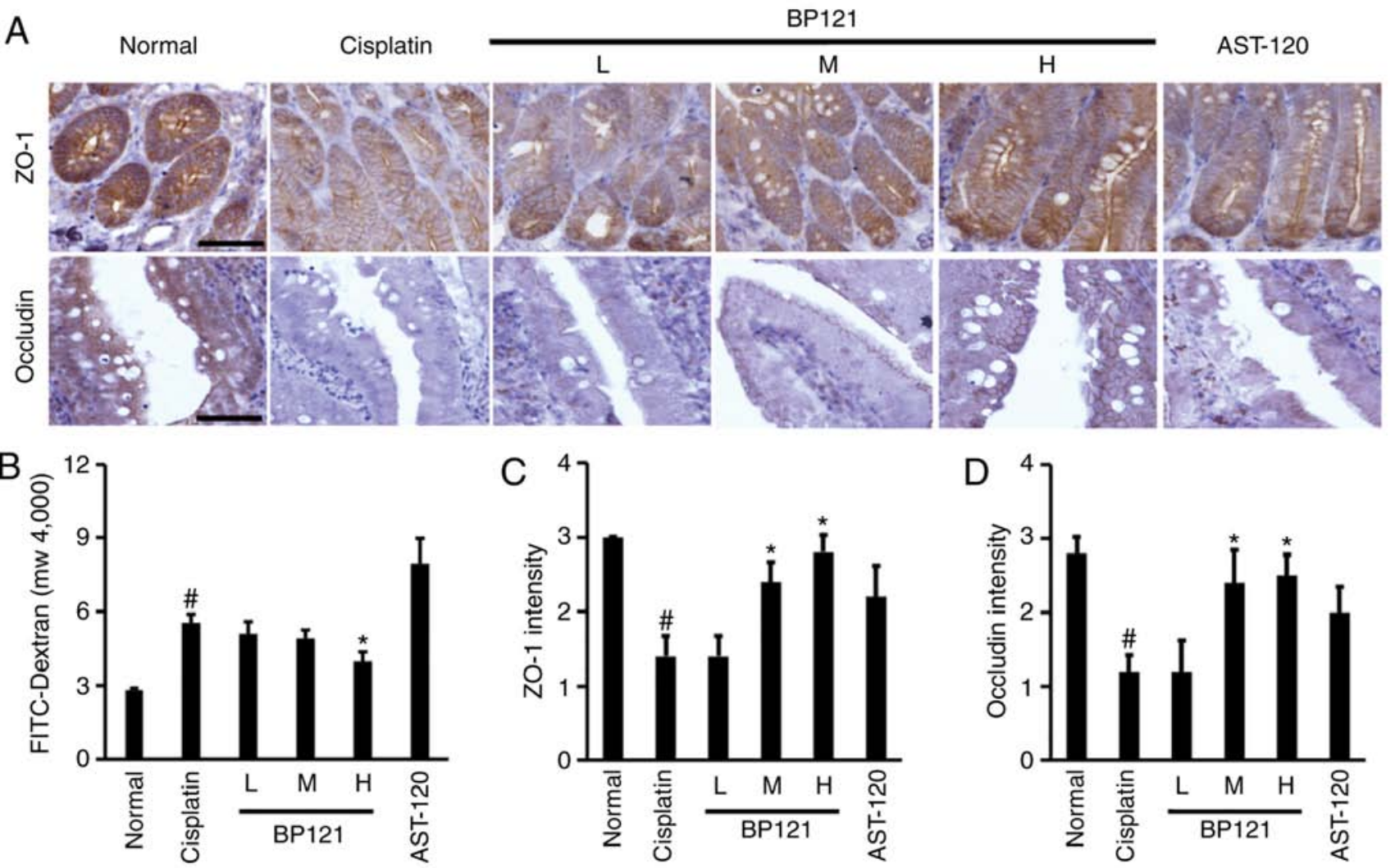

Figure 6. Histological evaluation of intestinal tissue in rats with cisplatin-induced acute kidney injury. (A) Immunohistochemical detection of ZO-1 and occludin. (B) Intestinal permeability as measured by FITC-dextran (MW=4,000 kDa). (C) Scoring of ZO-1 and (D) occludin immunoreactivity. Scale bar $=100 \mu \mathrm{m} .{ }^{*} \mathrm{P}<0.05$, normal vs. cisplatin; " $\mathrm{P}<0.05$, cisplatin vs. treatment group. FITC, fluorescein isothiocyanate; ZO, zona occludens.
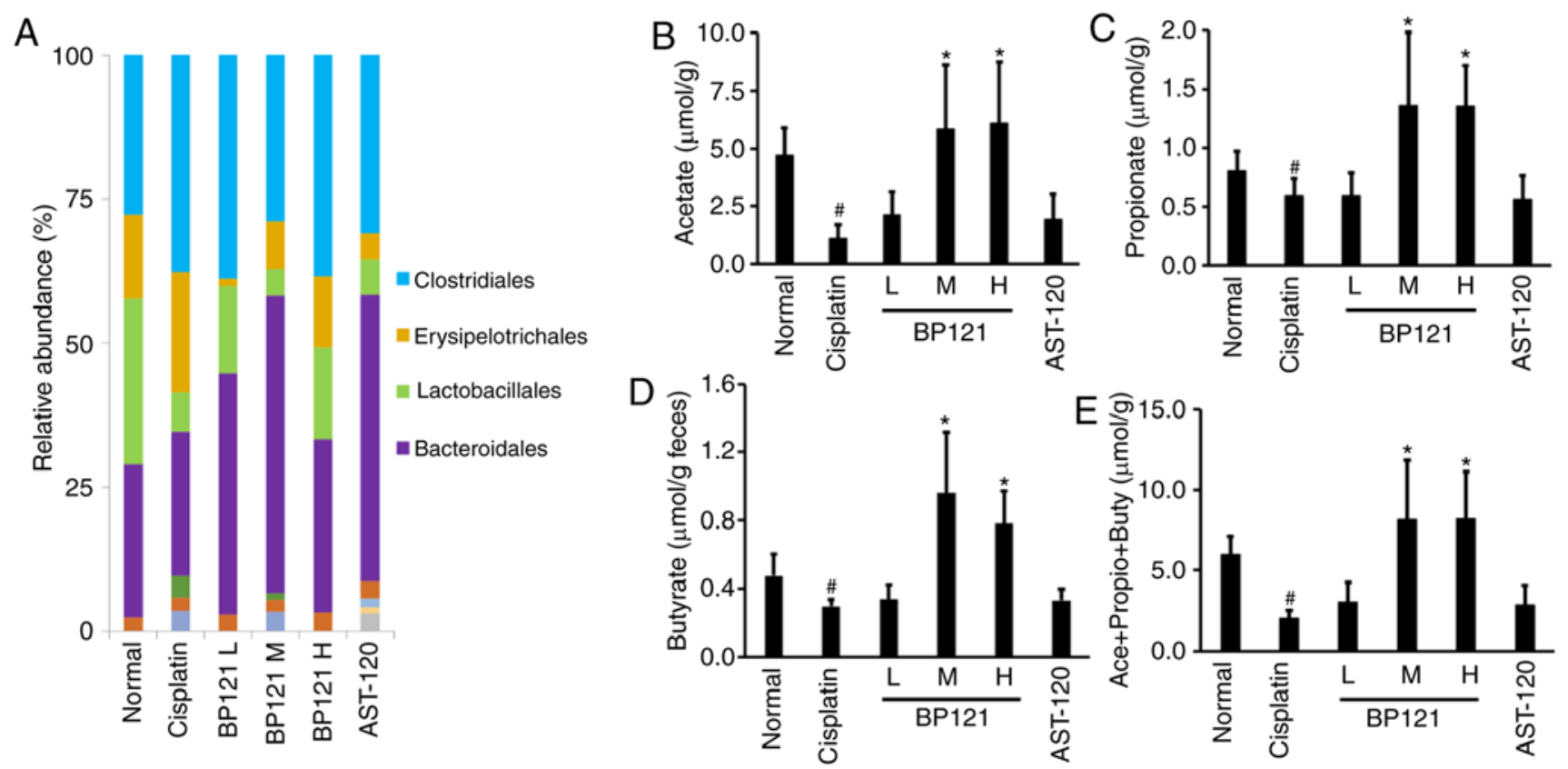

Figure 7. Change in microbiota abundance in the feces of rats with cisplatin-induced acute kidney injury following BP121 administration. (A) Relative abundance of gut microbiota under indicated conditions. (B) Acetate, (C) proprionate, (D) butyrate and (E) Ace+Propio+Buty concentrations in feces. ${ }^{*} \mathrm{P}<0.05$, normal vs. cisplatin; ${ }^{*} \mathrm{P}<0.05$, cisplatin vs. treatment group.

with medium and high concentrations of BP121 (Fig. 5D and E). No changes in oxidative stress relative to the cisplatin-treated group was observed upon AST-120 administration.
BP121 reverses cisplatin-induced increase in intestinal permeability. Intestinal permeability was increased by cisplatin; this was accompanied by downregulation of ZO-1 

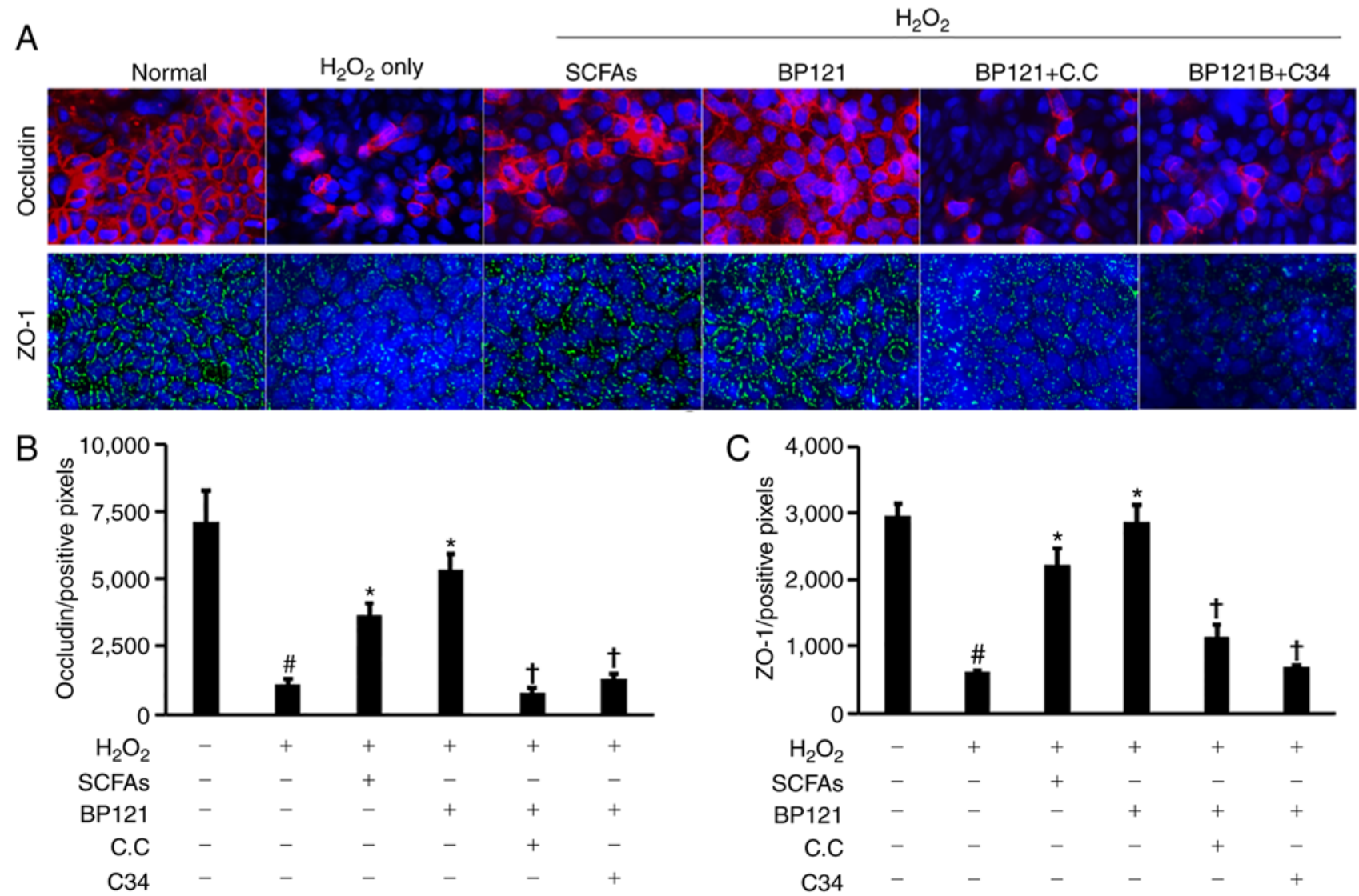

Figure 8. Expression of tight junction proteins in Caco-2 cells following $\mathrm{H}_{2} \mathrm{O}_{2}$ treatment. (A) Immunocytochemical detection of occludin and ZO-1 under indicated treatment conditions; nuclei were stained with DAPI. Quantitative analysis of (B) occludin and (C) ZO-1 immunoreactivity. BP121, treatment with BP121 culture supernatant for $48 \mathrm{~h}$; C.C, Compound C (AMPK inhibitor); C34, TLR4-IN-C34 (TLR4 inhibitor). ${ }^{\#} \mathrm{P}<0.05$, untreated vs. $\mathrm{H}_{2} \mathrm{O}_{2}$-treated; ${ }^{*} \mathrm{P}<0.05$, $\mathrm{H}_{2} \mathrm{O}_{2}$-treated vs. SCFA- and BP121 supernatant-treated; 'P $<0.05$, BP121 supernatant-treated vs. C.C- and C34-treated. SCFA, short chain fatty acids; ZO-1, zona occludens; TLR, toll-like receptor.

and occludin (Fig. 6A-D). However, medium and high doses of BP121 restored the levels of these proteins and reduced intestinal permeability. Treatment with AST 120 did not affect TJ protein expression or intestinal permeability.

BP121 causes changes in intestinal flora and stimulates SCFA production. The $16 \mathrm{~S}$ rRNA analysis showed that the proportion of Lactobacillales, a representative order of beneficial bacteria, was decreased by cisplatin; however, the abundance was restored upon BP121 administration. On the other hand, there were no changes in the proportion of Clostridiales, an order of harmful bacteria, across treatment groups (Fig. 7A). The concentrations of the representative SCFAs acetate, propionate and butyrate, which are known to regulate the expression of TJ proteins-in feces were examined and it was observed that they were decreased in cisplatin-treated rats. However, medium and high concentrations of BP121 increased the levels of all three SCFAs. AST-120 administration had no effect on SCFA levels (Fig. 7B-E).

BP121 increases TJ protein expression in Caco-2 cells. In vitro experiments were carried out using Caco-2 cells to clarify the mechanism by which BP121 improves intestinal permeability. $\mathrm{H}_{2} \mathrm{O}_{2}$ treated Caco-2 cells showed a marked decrease of both occludin and ZO-1 expression and pretreatment with SCFA or BP121 partially restored their expression. However, cotreatment with inhibitors of AMPK or TLR4 blocked the beneficial effect of BP121, suggesting that BP121 mediated barrier modulation is dependent on AMPK and TLR4 signaling (Fig. 8A-C).

\section{Discussion}

The human intestine contains over 1,000 species of bacteria that interact dynamically with the host, which plays an important role in health and disease (25). The microbiota is important for maintaining the integrity of the intestinal barrier as well as for immunological tolerance and metabolic activities that have not evolved in the host $(26,27)$. Intestinal dysbiosis is implicated in various pathological processes including inflammatory bowel disease, diabetes, obesity and AKI (27-29). Supplementing the gut microbiome with beneficial bacteria such as LAB can promote host health.

The present study investigated the effects of $L$. salivarius BP121, one of the 15 intestinal microorganisms isolated from the feces of newborns-on AKI. Previous studies have shown that the two-peptide bacteriocin ABP-118 produced by L. salivarius inhibits the growth of pathogenic bacteria but not most Lactobacillus species $(30,31)$. The current study speculated that $L$. salivarius could contribute to the treatment of diseases caused by dysbiosis. Indeed, the present results showed that BP121 increased the abundance of Lactobacillales in rats with cisplatin-induced dysbiosis. 
The intercellular junctional complex is an important component of the intestinal barrier. TJ proteins such as ZO-1 and occludin seal the paracellular space between epithelial cells (32), thus preventing the leakage of solutes such as microorganisms and antigens as they are transported across the epithelium (33). In this study, BP121 increased the expression of TJ proteins that were downregulated by $\mathrm{H}_{2} \mathrm{O}_{2}$ and cisplatin treatments. This is consistent with a previous study in which Lactobacillus rhamnosus GG increased the expression of human epithelial TJ proteins in vivo, which conferred protection against alcohol-induced epithelial barrier disruption (34). Moreover, L. plantarum administration increased ZO-1 and occludin levels in an animal model of alcoholic liver disease (34), and prevented the loss of TJ proteins and abolished the increase in epithelial permeability induced by phorbol ester in Caco-2 cells (35). Probiotics prevent damage to TJs by stimulating lipoxygenase activity via SCFAs (36). The maintenance of TJ proteins also involves activation of AMPK signaling $(34,35)$. In the present study, a 48-h treatment with BP121 culture supernatant containing SCFAs alleviated TJ protein damage caused by $\mathrm{H}_{2} \mathrm{O}_{2}$ in Caco- 2 cells; however, this effect was blocked by AMPK inhibitor. These results are in agreement with previous reports that SCFAs such as butyrate and acetate can preserve intestinal barrier integrity by SCFAs (37-39).

SCFAs are straight-chain saturated FAs with fewer than six carbon atoms; acetate, propionate and butyrate are the most abundant SCFAs in the human intestinal tract (11). SCFAs are the end products of fermentation of complex polysaccharides (40) including non-digestible dietary fibers such as inulin (41) and endogenous substrates such as epithelium-derived mucus (42). SCFAs are not only present in the gut, but can also be absorbed in the bloodstream (13) and protect against kidney injury by suppressing pro-inflammatory chemokines and cytokines such as IL- 6 , TNF- $\alpha$, and nitric oxide along with the production of reactive oxygen species (ROS) and apoptosis $(43,44)$, and/or by stimulating the production of the anti-inflammatory cytokine IL-10 (45) via $\mathrm{G}$ protein-coupled receptor activation (46) or histone deacetylase inhibition (47).

The antioxidant properties of probiotics have been confirmed and demonstrated by multiple studies $(48,49)$. ROS were shown to mediate cisplatin-induced AKI $(5,8)$ and it was demonstrated that $\mathrm{H}_{2} \mathrm{O}_{2}$ and $\mathrm{OH}$ radicals are involved in cisplatin-induced necrosis and apoptosis, respectively (49). In this study, BP121 decreased lipid peroxidation and increased the GSH/GSSG ratio; this was accompanied by reductions in the number of TUNEL- and Bax-positive cells and decreases in the levels of MCP-1 and the pro-inflammatory cytokines IL- 6 and TNF- $\alpha$ in kidney tissue.

IS and PCS are uremic toxins that are not efficiently removed by hemodialysis (50). They are generated from bacterial fermentation of tryptophan, aromatic amino acids and tyrosine followed by sulfation in the liver or colonic mucosa (50). In the plasma, IS and PCS are protein-bound and accumulate upon kidney failure (51). The concentrations of both toxins are correlated with progression of chronic kidney disease and higher risk of mortality (52). The observed increases in serum IS and PCS levels could result from enhanced production of these toxins, presumably due to an increase in the abundance of specific microbiota and disruption of the intestinal barrier by IS itself or by a decline in the Lactobacillales population (53). In the present study, treatment of AKI rats with BP121 stabilized intestinal barrier function and reduced serum levels of IS and PCS, which suppressed in renal inflammation.

In conclusion, the current results demonstrate that the newly isolated L. salivarius strain BP121 protects against cisplatin-induced AKI by suppressing IS and PCS production through modulation of the gut environment and regulation of TJ protein assembly via AMPK activation. These findings suggest that probiotics including BP121 are an effective treatment for dysbiosis and can prevent AKI.

\section{Acknowledgements}

Not applicable.

\section{Funding}

The present study was supported by Green Cross Wellbeing Corp (grant no. GCWB108).

\section{Availability of data and materials}

All data generated or analyzed in the present study are included in this published article.

\section{Authors' contributions}

THL, DP and YJK contributed to the study conception and design, as well as the data acquisition and analysis, interpretation. THL, DP drafted the manuscript. IL performed the immunohistochemical staining and in vitro studies. SK, CTO and JYK contributed to data interpretation. JY and SKJ contributed in study conception and reviewing the draft. All authors read and approved the final manuscript.

\section{Ethics approval and consent to participate}

The present study was approved by the Institutional Animal Care and Use Committee of Hoseo University [approval no. HSIACUC-18-097(1)].

\section{Patient consent for publication}

Not applicable.

\section{Competing interests}

The authors declare that they have no competing interests.

\section{References}

1. Zuk A and Bonventre JV: Acute kidney injury. Annu Rev Med 67: 293-307, 2016.

2. Bellomo R, Kellum JA and Ronco C: Acute kidney injury. Lancet 380: 756-766, 2012.

3. Hsu CY, McCulloch CE, Fan D, Ordonez JD, Chertow GM and Go AS: Community-based incidence of acute renal failure. Kidney Int 72: 208-212, 2007. 
4. Rahman M, Shad F and Smith MC: Acute kidney injury: A guide to diagnosis and management. Am Fam Physician 86: 631-639, 2012.

5. Ozkok A and Edelstein CL: Pathophysiology of cisplatin-induced acute kidney injury. Bio Med Res Int 2014: 967826, 2014.

6. Chen YY, Chen DQ, Chen L, Liu JR, Vaziri ND, Guo Y and Zhao YY: Microbiome-metabolome reveals the contribution of gut-kidney axis on kidney disease. J Transl Med 17: 5, 2019.

7. Gong J, Noel S, Pluznick JL, Hamad ARA and Rabb H: Gut microbiota-kidney cross-talk in acute kidney injury. Semin Nephrol 39: 107-116, 2019.

8. Pabla N and Dong Z: Cisplatin nephrotoxicity: Mechanisms and renoprotective strategies. Kidney Int 73: 994-1007, 2008.

9. Wang D and Lippard SJ: Cellular processing of platinum anticancer drugs. Nat Rev Drug Discov 4: 307-320, 2005.

10. Cohen SM and Lippard SJ: Cisplatin: From DNA damage to cancer chemotherapy. Prog Nucleic Acid Res Mol Biol 67: 93-130, 2001.

11. Joyce K, Saxena S, Williams A, Damurjian C, Auricchio N, Aluotto S, Tynan H and Demain AL: Antimicrobial spectrum of the antitumor agent, cisplatin. J Antibiot (Tokyo) 63: 530-532, 2010.

12. Taur Y and Pamer EG: Microbiome mediation of infections in the cancer setting. Genome Med 8: 40, 2016.

13. Andrade-Oliveira V, Amano MT, Correa-Costa M, Castoldi A Felizardo RJ, de Almeida DC, Bassi EJ, Moraes-Vieira PM, Hiyane MI, Rodas AC, et al: Gut bacteria products prevent AKI induced by ischemia-reperfusion. J Am Soc Nephrol 26 : $1877-1888,2015$.

14. Barrows IR, Ramezani A and Raj DS: Gut feeling in AKI: The long arm of short-chain fatty acids. J Am Soc Nephrol 26 : $1755-1757,2015$

15. LeBlanc JG, Chain F, Martín R, Bermúdez-Humarán LG, Courau S and Langella P: Beneficial effects on host energy metabolism of short-chain fatty acids and vitamins produced by commensal and probiotic bacteria. Microb Cell Fact 16: 79, 2017

16. Shimizu J, Kubota T, Takada E, Takai K, Fujiwara N, Arimitsu N Murayama MA, Ueda Y, Wakisaka S, Suzuki T and Suzuki N: Propionate-producing bacteria in the intestine may associate with skewed responses of IL10-producing regulatory T cells in patients with relapsing polychondritis. PLoS One 13: e0203657, 2018.

17. Parada Venegas D, De la Fuente MK, Landskron G, González MJ, Quera R, Dijkstra G, Harmsen HJM, Faber KN and Hermoso MA: Short chain fatty acids (SCFAs)-mediated gut epithelial and immune regulation and its relevance for inflammatory bowel diseases. Front Immunol 10: 277, 2019.

18. Chen T, Kim CY, Kaur A, Lamothe L, Shaikh M, Keshavarzian A and Hamaker BR: Dietary fibre-based SCFA mixtures promote both protection and repair of intestinal epithelial barrier function in a caco-2 cell model. Food Funct 8: 1166-1173, 2017.

19. Diao H, Jiao AR, Yu B, Mao XB and Chen DW: Gastric infusion of short-chain fatty acids can improve intestinal barrier function in weaned piglets. Genes Nutr 14: 4, 2019.

20. Meimandipour A, Hair-Bejo M, Shuhaimi M, Azhar K, Soleimani AF, Rasti B and Yazid AM: Gastrointestinal tract morphological alteration by unpleasant physical treatment and modulating role of lactobacillus in broilers. Br Poult Sci 51: $52-59,2010$.

21. Fujii H, Nishijima F, Goto S, Sugano M, Yamato H, Kitazawa R, Kitazawa S and Fukagawa M: Oral charcoal adsorbent (AST-120) prevents progression of cardiac damage in chronic kidney disease through suppression of oxidative stress. Nephrol Dial Transplant 24: 2089-2095, 2009.

22. Wang CY, Lin PR, Ng CC and Shyu YT: Probiotics properties of lactobacillus strains isolated from the feces of breast-fed infants and Taiwanese pickled cabbage. Anaerobe 16: 578-585, 2010.

23. Fang CY,Lu JR, Chen BJ, Wu C, Chen YP and Chen MJ: Selection of uremic toxin-reducing probiotics in vitro and in vivo. J Funct Foods 7: 407-415, 2014

24. McDonald JW and Pilgram TK: Nuclear expression of p53, p21 and cyclin D1 is increased in bronchioloalveolar carcinoma. Histopathology 34: 439-446, 1999.

25. Pagliari D, Piccirillo CA, Larbi A and Cianci R: The interactions between innate immunity and microbiota in gastrointestinal diseases. J Immunol Res 2015: 898297, 2015

26. Ley RE, Turnbaugh PJ, Klein S and Gordon JI: Microbial ecology: Human gut microbes associated with obesity. Nature 444 1022-1023, 2006.

27. Turnbaugh PJ and Gordon JI: The core gut microbiome, energy balance and obesity. J Physiol 587: 4153-4158, 2009.
28. Wang Y, Hoenig JD, Malin KJ, Qamar S, Petrof EO, Sun J, Antonopoulos DA, Chang EB and Claud EC: 16S rRNA gene-based analysis of fecal microbiota from preterm infants with and without necrotizing enterocolitis. ISME J 3: 944-954, 2009.

29. Jeffery IB, O'Toole PW, Öhman L, Clasesson MJ, Deane J, Quigley EM and Simre'n M: An irritable bowel syndrome subtype defined by species-specific alterations in faecal microbiota. Gut 61: 997-1006, 2012.

30. Barrett E, Hayes M, O'Connor P, Gardiner G, Fitzgerald GF, Stanton C and Hill C: Salivaricin P: One of a family of two-component antilisterial bacteriocins produced by intestinal isolates of Lactobacillus salivarius. Appl Environ Microbiol 73 . 3719-3723, 2007

31. Giongo A, Gano KA, Crabb DB, Mukherjee N, Novelo LL, Casella G, Drew JC, Ilonen J, Knip M, Hyöty H, et al: Toward defining the autoimmune microbiome for type 1 diabetes. ISME J 5: 82-91, 2011.

32. Ley RE, Peterson DA and Gordon JI: Ecological and evolutionary forces shaping microbial diversity in the human intestine. Cell 124: 837-848, 2006.

33. Wells JM, Rossi O, Meijerink M and van Baarlen P: Epithelial crosstalk at the microbiota-mucosal interface. Proc Natl Acad Sci USA 108(Suppl 1): 4607-4614, 2011

34. Bryniarski MA, Hamarneh F and Yacoub R: The role of chronic kidney disease-associated dysbiosis in cardiovascular disease. Exp Biol Med (Maywood) 244: 514-525, 2019.

35. Karczewski J, Troost FJ, Konings I, Dekker J, Kleerebezem M, Brummer RJM and Wells JM: Regulation of human epithelial tight junction proteins by Lactobacillus plantarum in vivo and protective effects on the epithelial barrier. Am J Physiol Gastrointest Liver Physiol 298: G851-G859, 2010.

36. Vaziri ND: CKD impairs barrier function and alters microbial flora of the intestine: A major link to inflammation and uremic toxicity. Curr Opin Nephrol Hypertens 21: 587-592, 2012.

37. Mariadason JM, Barkla DH and Gibson PR: Effect of short-chain fatty acids on paracellular permeability in Caco-2 intestinal epithelium model. Am J Physiol 272: G705-G712, 1997.

38. Kendle M and Maslowski: The role of GPR43 in the immune system: A novel connection between diet, gut microbiota and immune function. University of New South Wales, Sydney, 2013

39. Nyman M: Fermentation and bulking capacity of indigestible carbohydrates: The case of inulin and oligofructose. Br J Nutr 87 (Suppl 2): 163-168, 2002.

40. Kotzampassi K, Giamarellos-Bourboulis EJ and Stavrou G: Obesity as a consequence of gut bacteria and diet interactions. ISRN Obes 2014: 651895, 2014

41. Wong JM, de Souza R, Kendall CW, Emam A and Jenkins DJ: Colonic health: Fermentation and short chain fatty acids. J Clin Gastroenterol 40: 235-243, 2006.

42. Pomare EW, Branch WJ and Cummings JH: Carbohydrate fermentation in the human colon and its relation to acetate concentrations in venous blood. J Clin Invest 75: 1448-1454, 1985.

43. Kim MH, Kang SG, Park JH, Yanagisawa M and Kim CH: Short-chain fatty acids activate GPR41 and GPR43 on intestinal epithelial cells to promote inflammatory responses in mice. Gastroenterology 145: 396-406, 2013.

44. Tedelind $S$, Westberg $F$, Kjerrulf $M$ and Vidal $A$ Anti-inflammatory properties of the short-chain fatty acids acetate and propionate: A study with relevance to inflammatory bowel disease. World J Gastroenterol 13: 2826-2832, 2007.

45. Liu T, Li J, Liu Y, Xiao N, Suo H, Xie K, Yang C and Wu C: Short-chain fatty acids suppress lipopolysaccharide-induced production of nitric oxide and proinflammatory cytokines through inhibition of NF- $\kappa$ B pathway in RAW264.7 cells. Inflammation 35: 1676-1684, 2012.

46. Tazoe H, Otomo Y, Karaki S, Kato I, Fukami Y, Terasaki M and Kuwahara A: Expression of short-chain fatty acid receptor GPR41 in the human colon. Biomed Res 30: 149-156, 2009.

47. Wang Y, Wu Y, Wang Y, Xu H, Mei X, Yu D and Li W: Antioxidant properties of probiotic bacteria. Nutrients 9: 521-535, 2017.

48. Mishra V, Shah C, Mokashe N, Chavan R, Yadav H and Prajapati J: Probiotics as potential antioxidants: A systematic review. J Agric Food Chem 63: 3615-3626, 2015.

49. Baek SM, Kwon CH, Kim JH, Woo JS, Jung JS and Kim YK: Differential roles of hydrogen peroxide and hydroxyl radical in cisplatin-induced cell death in renal proximal tubular epithelial cells. J Lab Clin Med 142: 178-186, 2003. 
50. Meijers BK and Evenepoel P: The gut-kidney axis: Indoxyl sulfate, p-cresyl sulfate and CKD progression. Nephrol Dial Transplant 26: 759-761, 2011.

51. Jourde-Chiche N, Dou L, Cerini C, Dignat-George F, Vanholder R and Brunet P: Protein-bound toxins-update 2009. Semin Dial 22: 334-339, 2009.

52. Ramezani A and Raj DS: The gut microbiome, kidney disease, and targeted interventions. J Am Soc Nephrol 25: 657-670, 2014.
53. Yoshifuji A, Wakino S, Irie J, Tajima T, Hasegawa K, Kanda T, Tokuyama $\mathrm{H}$, Hayashi $\mathrm{K}$ and Itoh $\mathrm{H}$ : Gut Lactobacillus protects against the progression of renal damage by modulating the gut environment in rats. Nephrol Dial Transplant 31: 401-412, 2015.

c) (i) () This work is licensed under a Creative Commons Attribution-NonCommercial-NoDerivatives 4.0 International (CC BY-NC-ND 4.0) License. 